\title{
2-SPHERE BUNDLES OVER COMPACT SURFACES
}

\author{
PAUL MELVIN ${ }^{1}$
}

\begin{abstract}
Closed 4-manifolds which fiber over a compact surface with fiber a sphere are classified, and the fibration is shown to be unique (up to diffeomorphism).
\end{abstract}

It is well known that there are at most two orientable 4-manifolds which fiber over a given compact surface with fiber the 2-sphere $S^{2}$. (There is exactly one if the surface has nonempty boundary, and two if it is closed.) If the orientability condition is dropped, then the situation becomes more involved. In particular the $(\bmod 2)$ intersection pairing is no longer sufficient to distinguish among the manifolds that arise. One must also consider the $\pi_{1}$-action on $\pi_{2}$ and the peripheral structure.

The purpose of this note is to classify all 4-manifolds (orientable or not) which are total spaces of $S^{2}$-bundles over compact surfaces. We shall work in the smooth category. Since $\operatorname{Diff}\left(S^{2}\right)$ deformation retracts to $O(3)$, we may assume that all bundles that arise have $O(3)$ as structure group.

Along the way it is shown that the bundle structures are unique. That is, if any two 4-manifolds, fibered as above, are diffeomorphic, then there is a fiber preserving diffeomorphism between them which is orthogonal on fibers.

Our interest in $S^{2}$-bundles arose in the study of Lie group actions (in particular of $S O(3)$ ) on 4-manifolds. The results obtained here are used in the equivariant classification of such actions [MP].

1. Classification. In this section we establish notation and state our results. Proofs are deferred to the next section.

Throughout the paper, all homology and cohomology groups will have coefficients in $\mathbf{Z}_{2}$.

First observe that to classify the 4-manifolds of the title, one may consider one surface at a time.

Proposition. No 4-manifold is the total space of two different $S^{2}$-bundles over distinct compact surfaces.

Received by the editors November 3, 1983.

1980 Mathematics Subject Classification. Primary 55R25, 57R22, 57S15.

${ }^{1}$ Supported in part by National Science Foundation Grant MCS82-05450. 
Hence we fix $F$, a compact connected 2-dimensional surface, and consider $S^{2}$-bundles

$$
\xi: \begin{array}{cc}
M^{4} & \\
\downarrow & p \\
& F
\end{array}
$$

with structure group $O(3)$. Write $w_{i}(\xi)$ for the Stiefel-Whitney classes of $\xi$ in $H^{i}(F)$ (recall that coefficients are in $\mathbf{Z}_{2}$ ), and $w(\xi)$ for the total Stiefel-Whitney class.

Bundle theory shows that $\xi$ is classified up to bundle equivalence by $w(\xi)$. In particular one inay construct $M$, knowing $w(\xi)$, as follows (we omit the proof):

STRUCTURE Lemma. To construct the total space $M$ of the bundle $\xi$ (above), start with $F \times S^{2}$ and a properly embedded 1-manifold $\omega$ in $F$ representing the Poincare dual of $w_{1}(\xi)$. Then

(1) cut $F \times S^{2}$ along $\omega \times S^{2}$ and reidentify opposite $S^{2}$ fibers along the cut by the antipodal map, and

(2) if $w_{2}(\xi) \neq 0$ ( $F$ necessarily closed), then also cut along $\partial D \times S^{2}$ (where $D$ is a 2-disc in $F$ ) and reidentify opposite fibers by using the diffeomorphism of $\partial D \times S^{2}$ coming from the nontrivial element of $\pi_{1}(S O(3))$.

Observe that distinct bundles may have diffeomorphic total spaces. For example any diffeomorphism $h: F \rightarrow F$ induces a diffeomorphism between the total spaces of $\xi$ and the pull back $h^{*} \xi$, whereas $w(\xi)$ need not equal $w\left(h^{*} \xi\right)=h^{*}(w(\xi))$. It turns out that this example is generic:

THEOREM 1. Let

$$
\xi: \begin{gathered}
M \\
\downarrow
\end{gathered} \quad p \quad \text { and } \xi^{\prime}: \begin{array}{cc}
M^{\prime} \\
\downarrow \\
F
\end{array}
$$

be two $S^{2}$-bundles over a compact surface $F$. Then $M$ and $M^{\prime}$ are diffeomorphic if and only if there is a diffeomorphism $h: F \rightarrow F$ with $\xi=h^{*}\left(\xi^{\prime}\right)$ (i.e. $\xi$ and $\xi^{\prime}$ are weakly equivalent ).

To give an explicit classification of the total spaces that arise, one needs a classification of $S^{2}$-bundles over $F$ up to weak equivalence.

THEOREM 2. With the hypothesis of Theorem $1, \xi$ and $\xi^{\prime}$ are weakly equivalent if and only if $w_{1}(\xi) \sim w_{1}\left(\xi^{\prime}\right)$ and $w_{2}(\xi)=w_{2}\left(\xi^{\prime}\right)$, where $\sim$ is the equivalence relation defined below.

Definition. Let $w$ be a class in $H^{1}(F)$. Define two invariants, $r(w)$ (a nonnegative even integer) and $s(w)(=0,1,2$ or $\infty)$, as follows:

Set $r(w)$ equal to the number of components of $\partial F$ to which $w$ restricts nontrivially. (Dually, if $\omega$ is a 1-manifold in $F$ representing the Poincaré dual of $w$, then $r(w)$ is the number of components of $\partial F$ which contain an odd number of points of $\partial \omega$.

Set $s(w)=0$ if $r(w)>0$. If $r(w)=0$ then consider two cases: $F$ closed. If $w=w_{1}(F)$ (the first tangential Stiefel-Whitney class of $F$ ) then set $s(w)=\infty$. If 
$w=0 \neq w_{1}(F)\left(F\right.$ necessarily nonorientable) then set $s(w)=0$. If $w \neq 0$ or $w_{1}(F)$, then set $s(w)=1$ or 2 , respectively, according to whether $w \cup w_{1}(F)=1$ or 0 (or dually whether $\omega$ is orientation reversing or preserving). $F$ bounded. Let $\hat{F}$ be the closed surface obtained by capping off $F$ along $\partial F$ with 2-discs. Consider the exact sequence

$$
0 \rightarrow H^{1}(\hat{F}) \rightarrow H^{1}(F) \rightarrow H^{2}(\hat{F}, F) \rightarrow
$$

of the pair $(\hat{F}, F)$. Since $r(w)=0$, the image of $w$ in $H^{2}(\hat{F}, F)$ is zero. So $w$ is the restriction of a unique class $\hat{w}$ in $H^{1}(\hat{F})$. (Dually, $\omega$ may be chosen to be a closed curve in $F$, and $\hat{w}$ is the Poincare dual of the homology class represented by this curve in $\hat{F})$. Set $s(w)=s(\hat{w})$.

Now for $w$ and $w^{\prime}$ in $H^{1}(F)$, define $w \sim w^{\prime}$ if and only if (I) $r(w)=r\left(w^{\prime}\right)$, and (II) $s(w)=s\left(w^{\prime}\right)$. This relation is designed so that $w \sim w^{\prime}$ if and only if there is a diffeomorphism $h: F \rightarrow F$ with $w=h^{*}\left(w^{\prime}\right)$ (see Lemma 2 in the next section).

REMARK. The referee observed that for the bundles under consideration, the structure group reduces to $O(2)$. A classification of $S^{1}$-bundles over $F$ with group $O(2)$ was given by Seifert [S] and Orlik-Raymond [OR] for $F$ closed, and by Fintushel $[\mathbf{F}]$ for $F$ with boundary. The invariants used are the same as ours (in the notation of $[\mathbf{F}], k$ corresponds to $r$ and $\varepsilon$ to $s$ ). Theorem 2 follows easily. For completeness, we shall give an independent proof.

It follows from Theorems 1 and 2 that the total space $M$ of the bundle $\xi$ is classified up to diffeomorphism by the invariants $r\left(w_{1}(\xi)\right), s\left(w_{1}(\xi)\right)$ and $w_{2}(\xi)$. For closed base surfaces $F, r\left(w_{1}(\xi)\right)=0, w_{2}(\xi)=0$ or 1 , and $s\left(w_{1}(\xi)\right)$ can assume any of the following values:

\begin{tabular}{|l|l|}
\multicolumn{1}{l|}{ closed $F$} & $s\left(w_{1}(\xi)\right)$ \\
\hline$S^{2}$ & $\infty$ \\
orientable $\neq S^{2}$ & $2, \infty$ \\
$P^{2}$ (projective plane) & $0, \infty$ \\
$K^{2}$ (Klein bottle) & $0,1, \infty$ \\
nonorientable $\neq P^{2}$ or $K^{2}$ & $0,1,2, \infty$ \\
\hline
\end{tabular}

Thus there are two $S^{2}$-bundles over $S^{2}$, up to diffeomorphism, four over each orientable surface $\neq S^{2}$ and over $P^{2}$, six over $K^{2}$, and eight over each nonorientable surface $\neq P^{2}$ or $K^{2}$. If $F$ is bounded, then $w_{2}(\xi)=0$ but $r\left(w_{1}(\xi)\right)$ may be any nonnegative even integer $\leqslant \operatorname{dim} H_{0}(\partial F)$. For example, there are $3+[k / 2] S^{2}$ bundles over the $k$-punctured Klein bottle, up to diffeomorphism.

\section{Proofs.}

Proof of the Proposition. Suppose that a given 4-manifold $M$ fibers over a compact surface $F$ with fiber $S^{2}$. Evidently $M$ and $F$ have the same number of boundary components. By the homotopy sequence of a fibration, $\pi_{1}(F)=\pi_{1}(M)$. Furthermore, if $M$ has nonempty boundary then the double $D M$ of $M$ is an $S^{1}$-bundle over the double $D F$ of $F$, and so $\pi_{1}(D F)=\pi_{1}(D M)$. Thus from $M$ one may compute $\pi_{1}(F), \pi_{1}(D F)$, and the number of components of $\partial F$. It follows from the classification of surfaces that $F$ is uniquely determined by $M$. 
We shall use two lemmas in the proofs of Theorems 1 and 2.

LEMMA 1. Let

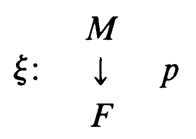

be an $S^{2}$-bundle over a compact surface $F$. Then $p^{*}: H^{*}(F) \rightarrow H^{*}(M)$ is injective and $w(M)=p^{*}(w(F) \cup w(\xi))$ (where $w(M)$ and $w(F)$ are the total tangential StiefelWhitney classes $)$. In particular

(a) $w_{1}(M)=p^{*}\left(w_{1}(F)+w_{1}(\xi)\right)$,

(b) $w_{2}(M)=p^{*}\left(w_{2}(F)+w_{1}(F) \cup w_{1}(\xi)+w_{2}(\xi)\right)$.

Proof. The injectivity of $p^{*}$ follows from the Gysin sequence

$$
\rightarrow H^{*-3}(F) \rightarrow H^{*}(F) \stackrel{p^{*}}{\rightarrow} H^{*}(M) \rightarrow
$$

of $\xi$. To obtain the formula for $w(M)$, note that by definition $w(\xi)=w(\Xi)$, where $\Xi$ is the $B^{3}$-bundle $q: V^{5} \rightarrow F$ associated to $\xi$. Applying the Whitney product theorem to $\tau_{F} \oplus \Xi=\tau_{V \mid F}(\tau$ denotes tangent bundle $)$ gives

$$
w(V)=q^{*}(w(F) \cup w(\xi))
$$

and to $\tau_{M} \oplus \varepsilon=\tau_{V \mid M}$ gives $w(M)=i^{*} w(V)$ where $i: M \rightarrow V$ is inclusion. Since $p=q i$, the lemma follows.

LEMMA 2. Let $w$ and $w^{\prime}$ be classes in $H^{2}(F)$. Then there is a diffeomorphism $h: F \rightarrow F$ with $w=h^{*}\left(w^{\prime}\right)$ if and only if $w \sim w^{\prime}$.

Recall that $w \sim w^{\prime}$ if and only if (I) $r(w)=r\left(w^{\prime}\right)$, and (II) $s(w)=s\left(w^{\prime}\right)$ (see the previous section). Observe that condition II can be replaced by

(II) If $r(w)=0$ then

(i) $w=w^{\prime}$ if $w=0$ or $w_{1}(F)$,

(ii) $\hat{w} \cup w_{1}(\hat{F})=\hat{w}^{\prime} \cup w_{1}(\hat{F})$, where $\hat{F}=F$ and $\hat{w}=w$ if $F$ is closed.

Proof of Lemma 2 (CF. [F]). First assume that $h$ exists. Then $h^{*}\left(w_{1}(F)\right)=w_{1}(F)$, and if $F$ is bounded then $h$ extends to a diffeomorphism $\hat{h}: \hat{F} \rightarrow \hat{F}$ with $h^{*}\left(\hat{w}^{\prime}\right)=\hat{w}$. It follows readily from the definition of $\sim$ that $w \sim w^{\prime}$.

Conversely, assume that $w \sim w^{\prime}$. We may also assume that $w\left(\right.$ and $\left.w^{\prime}\right) \neq w_{1}(F)$ or 0 (in those cases take $h=$ identity).

Case 1: $F$ closed. Represent the Poincaré duals of $w$ and $w^{\prime}$ by embedded loops $\omega$ and $\omega^{\prime}$ with open tubular neighborhoods $W$ and $W^{\prime}$. Since $w \neq 0, W$ is nonseparating and so $F-W$ is connected. Since $w \neq w_{1}(F), F-W$ and $F$ have the same orientability (they are either both orientable or both nonorientable). The same argument for $w^{\prime}$ shows that $F-W$ and $F-W^{\prime}$ have the same orientability and are both connected. Since $w \cup w_{1}(F)=w^{\prime} \cup w_{1}(F)$, they also have the same number of boundary components. By the classification of compact surfaces, $F-W \cong F-W^{\prime}$, and so there is a diffeomorphism $h: F \rightarrow F$ carrying $\omega$ to $\omega^{\prime}$. Thus $h^{*}\left(w^{\prime}\right)=w$. 
Case 2: $F$ bounded. Set $r=r(w)=r\left(w^{\prime}\right)$. If $r=0$ then use the argument in Case 1. If $r>0$, then the Poincaré dual of $w$ (or $w^{\prime}$ ) can be represented by $r / 2$ properly embedded arcs $\omega$ (or $\left.\omega^{\prime}\right)$. The rest of the argument proceeds essentially as in Case 1 . That $F-W$ and $F$ have the same orientability follows from the fact that $\omega$ has a minimal number of components among relative cycles dual to $w$. Minimality also assures that all the points of $\partial \omega$ lie in distinct components of $W$, whence $F-W$ has exactly $r / 2$ fewer boundary components than does $F$.

For the proofs of Theorems 1 and 2, we adopt the following notation: $w_{i}=w_{i}(\xi)$ and $w_{i}^{\prime}=w_{i}\left(\xi^{\prime}\right)(i=1,2), r=r\left(w_{1}\right), s=s\left(w_{1}\right), r^{\prime}=r\left(w_{1}^{\prime}\right), s^{\prime}=s\left(w_{1}^{\prime}\right)$. We begin with Theorem 2.

Proof of Theorem 2. If $\xi$ and $\xi^{\prime}$ are weakly equivalent, then by definition there is a diffeomorphism $h: F \rightarrow F$ with $\xi=h^{*}\left(\xi^{\prime}\right)$. It follows that $w_{i}=h^{*}\left(w_{i}^{\prime}\right)(i=1,2)$. Thus $w_{1} \sim w_{1}^{\prime}$ (by Lemma 2) and $w_{2}=w_{2}^{\prime}$ (since $h^{*}$ is the identity on $H^{2}(F)$ ).

Conversely, assume that $w_{1} \sim w_{1}^{\prime}$ and $w_{2}=w_{2}^{\prime}$. By Lemma 2, there is a diffeomorphism $h: F \rightarrow F$ with $w_{1}=h^{*}\left(w_{1}^{\prime}\right)$. Since $h^{*}$ is the identity on $H^{2}(F), w_{2}=h^{*}\left(w_{2}^{\prime}\right)$ as well. It follows that $\xi=h^{*}\left(\xi^{\prime}\right)$.

Proof of TheOREM 1. First we make four assertions about the bundle $\xi$ (or $\xi^{\prime}$ ).

(1) $r$ is the number of nonorientable boundary components of $M$.

(2) (For $F \neq P^{2}$ ) $w_{1}=0$ if and only if $\pi_{1}(M)$ acts trivially on $\pi_{2}(M)$.

(3) $w_{1}=w_{1}(F)$ if and only if $w_{1}(M)=0$.

(4) $w_{2}=0$ if and only if the self-intersections of all classes in $H_{2}(M)$ are zero.

Assertions (1) and (2) follow from the structure lemma. (For (2) observe that if $F \neq S^{2}$ or $P^{2}$ then $\pi_{2}(M)=\mathbf{Z}$, generated by a fiber.) Lemma 1(a) gives (3). To prove (4), observe that there is an isomorphism $H_{2}(M) \cong H_{2}(F) \oplus H_{2}\left(S^{2}\right)$ (for example from the Leray-Hirsch Theorem). Thus $H_{2}(M)$ is generated by a section (if $F$ is closed) and a fiber of $\xi$. By the structure lemma, the section has zero self-intersection $(\bmod 2)$ if and only if $w_{2}=0$. Since the fiber has zero self-intersection, (4) follows.

Now assume that $M$ and $M^{\prime}$ are diffeomorphic. It suffices to show that $w_{1} \sim w_{1}^{\prime}$ and $w_{2}=w_{2}^{\prime}$, by Theorem 2 .

Case 1: $F$ closed. By assertion (4), $w_{2}=w_{2}^{\prime}$, since $H^{2}(F)=\mathbf{Z}_{2}$. Note that $r=0=r^{\prime}$ since $F$ is closed. Assertion (3) shows that $w_{1}=w_{1}^{\prime}$ if $w_{1}=w_{1}(F)$. This implies that $w_{1} \sim w_{1}^{\prime}$ for $F=P^{2}$, and so we assume $F \neq P^{2}$. Then by assertion (2), $w_{1}=w_{1}^{\prime}$ if $w_{1}=0$, establishing condition II(i) in the definition of $\sim$. It remains to verify that $w_{1} \cup w_{1}(F)=w_{1}^{\prime} \cup w_{1}(F)$ (condition II(ii)). But this is immediate from Lemma 1(b) since $w_{2}=w_{2}^{\prime}$ and $p^{*}$ is injective. Thus $w_{1} \sim w_{1}^{\prime}$.

Case 2: $F$ bounded. Assertion (1) gives $r=r^{\prime}$. It remains to show $s=s^{\prime}$. We may assume $r=0$. Recall that $s=s\left(\hat{w}_{1}\right)$, where $\hat{w}_{1}$ is the unique extension of $w_{1}$ to a class in $H^{1}(\hat{F})(\hat{F}=F$ capped off). Now $M$ can be capped off along $\partial M$ with copies of $B^{2} \times S^{2}$ to get an $S^{2}$-bundle $\hat{\xi}$ over $\hat{F}$ with $i^{*}(\hat{\xi})=\xi$ (where $i: F \rightarrow \hat{F}$ is inclusion) and $w_{2}(\hat{\xi})=0$. Then $w_{1}(\hat{\xi})=\hat{w}_{1}$. Let $\hat{M}$ be the total space of $\hat{\xi}$. A similar construction gives $\hat{\xi}^{\prime}$ with total space $\hat{M}^{\prime}$ diffeomorphic to $\hat{M}$. By Case $1, s\left(w_{1}(\hat{\xi})\right)=s\left(w_{1}\left(\hat{\xi}^{\prime}\right)\right)$, and so $s\left(\hat{w}_{1}\right)=s\left(\hat{w}_{1}^{\prime}\right)$. Thus $s=s\left(\hat{w}_{1}\right)=s\left(\hat{w}_{1}^{\prime}\right)=s^{\prime}$. This proves the bounded case.

The converse is trivial. 
REMARKS. (1) Theorems 1 and 2 can be obtained without reference to the tangent bundle of $M$, instead using a more delicate analysis of the $\pi_{1}(M)$-action on $\pi_{2}(M)$. Our approach shortens the proof.

(2) Theorems 1 and 2 can also be generalized to all higher dimensions, that is to $S^{n}$-bundles over compact surfaces with group $G=O(n+1)$. (If $G=\operatorname{PL}\left(S^{n}\right)$ then the resulting classification still holds in the PL category, by results of Browder [B] and Lashof-Shaneson [LS] on PL automorphisms of $S^{n} \times S^{1}$. What happens if $\left.G=\operatorname{Diff}\left(S^{n}\right) ?\right)$

\section{REFERENCES}

[B] W. Browder, Diffeomorphisms of 1-connected manifolds, Trans. Amer. Math. Soc. 128 (1967), 155-163.

[F] R. Fintushel, Local $S^{1}$-actions on 3-manifolds, Pacific J. Math. 66 (1976), 111-118.

[LS] R. K. Lashof and J. L. Shaneson, Classification of knots in codimension two, Bull. Amer. Math. Soc. 75 (1969), 171-175.

[MP] P. Melvin and J. Parker, 4-manifolds with large symmetry groups (to appear).

[OR] P. Orlik and F. Raymond, On 3-manifolds with local SO(2)-action, Quart. J. Math. 20 (1969), 143-160.

[S] H. Seifert, Topologie dreidimensionaler gefaseter Räume, Acta Math. 60 (1933), 147-238.

Department of Mathematics, Bryn Mawr College, Bryn Mawr, Pennsylvania 19010 